EPiC Series in Engineering
Volume 3, 2018, Pages 1568-1574
HIC 2018. 13th International
Conference on Hydroinformatics

\title{
Assessment on Effects of Preliminary Release Operation of a Multi-purpose Reservoir Considering Ensemble Inflow Prediction
}

\author{
Daisuke Nohara ${ }^{1 *}$ and Hiroki Saito ${ }^{2}$ \\ ${ }^{1}$ Disaster Prevention Research Institute, Kyoto University, Japan \\ ${ }^{2}$ Graduate School of Engineering, Kyoto University, Japan \\ nohara.daisuke.2v@kyoto-u.ac.jp
}

\begin{abstract}
A Monte Carlo-based method to analyze effectiveness and risks of integrated operation of a multi-purpose reservoir for flood management considering real-time ensemble hydrological predictions is developed in this study. Preliminary release operation, in which water stored in the reservoir is released just in advance of a flood event considering real-time hydrological predictions, is considered as an integrated reservoir operation method. A simulated generation method of ensemble hydrological predictions with a certain error structure is developed in order to generate a number of ensemble hydrological predictions for Monte Carlo simulation. A number of simulations of reservoir operation are conducted with consideration of the generated ensemble hydrological predictions to analyze the effects of preliminary release operation based on ensemble hydrological predictions. The result of the analysis can provide reservoir managers with quantitative and science-based information on expected benefits and risks to introduce operational hydrological predictions into reservoir operation from the long-term viewpoint.
\end{abstract}

\section{Introduction}

Reservoirs can play a significant role in water resources management to mitigate impacts of waterrelated disasters such as floods or droughts. More integrated operation of multi-purpose reservoirs, which usually have storage capacities for both flood control and water use purposes, is especially important for more effective management of those water-related disasters. Preliminary release operation, in which water storage in the reservoir is released just in advance of flood arrival considering real-time flood predictions to secure increased empty storage volume for flood control,

\footnotetext{
${ }^{*}$ Corresponding author
} 
can be considered as an effective way to derive more capability of a multi-purpose reservoir for both flood management and water supply.

As it is required for preliminary release operation of a reservoir to consider future hydrological conditions in the target river basin, consideration of real-time hydrological prediction is inevitable in this operation. Various operational hydrological predictions which have been provided usually by meteorological agencies can be good information to make a decision on preliminary release operation. Ensemble hydrological forecasts, which consist of multiple numerical prediction sequences (called as ensemble prediction members), are included in those predictions. Those numerical predictions can be estimated with different initial conditions or parameter settings of the numerical model in order to consider uncertainty in the prediction due to imperfect estimation of initial conditions or model parameters. One can therefore derive not only predicted values but also information on the degree of prediction uncertainty from the ensemble prediction by considering spread of the ensemble prediction members at each predicted time cross section. Thus, consideration of ensemble hydrological predictions is expected to contribute to more robust decision making in reservoir operation including preliminary release operation.

From this point of view, various studies have been carried out for application of ensemble hydrological predictions into real-time reservoir operation (Faber and Stedinger, 2001; Kim et al., 2007; Masuda and Oishi, 2013). These studies were mainly conducted to investigate the effectiveness of reservoir operation considering real-time ensemble hydrological predictions from case studies with real prediction data. However, a number of prediction data are needed to analyse the long-term effectiveness or risk to consider real-time hydrological predictions in order to rule out the impact of the randomness in prediction errors.

Considering these circumstances, a method to analyse effectiveness or risks of preliminary release operation of a multi-purpose reservoir considering ensemble hydrological predictions is developed. A Monte Carlo simulation of preliminary release operation of a reservoir is conducted for assessment of effects of reservoir operation when predictions are applied, considering an artificial generation process of ensemble inflow predictions with a designed error structure.

\section{Methodology}

\subsection{Framework of the Assessment on Effectiveness of Preliminary Release Operation}

The proposed framework for the assessment of effectiveness and risks of preliminary release operation of a multi-purpose reservoir considering ensemble inflow predictions can be summarized as follows. Firstly, error characteristics of ensemble predictions is determined to artificially generate ensemble inflow predictions with a certain error structure. One can derive error characteristics from operational ensemble hydrological predictions if available, in order to generate a number of ensemble inflow predictions with the same error structure of operational predictions. As error characteristics of ensemble predictions, the ensemble mean error and spread of the ensemble prediction is considered in this study as a fundamental analysis. A number of ensemble inflow predictions are then artificially generated by use of a simulated generation model of ensemble predictions considering those error parameters in order to supplement scarce prediction data provided for the same hydrological event with the same error structure. A simulation of preliminary release of a reservoir is respectively conducted for the target flood events considering generated ensemble inflow predictions as a part of a Monte Carlo simulation. The results of simulations are analysed in an integrated manner to analyse long-term effects to introduce preliminary release operation considering ensemble inflow predictions with the designed error structure. 


\subsection{Simulated Generation of Ensemble Inflow Predictions}

Ensemble inflow predictions are artificially generated to supplement scarcity of actual prediction data. The synthetic generation model developed by Nohara et al. (2017) is employed to generate ensemble inflow predictions in this study. In this method, a predicted value is generated by adding a value of prediction error that is artificially generated to the true value of inflow. Values of prediction error are generated by randomly sampling values from a probabilistic distribution which prediction errors are assumed to follow. Although one can assume several types of distributions such as normal distributions and $\log$ normal distributions as the candidate for the probabilistic distribution of prediction errors, a normal distribution is employed in this study for a fundamental case study of impact assessment on preliminary release operation considering ensemble predictions. Values of prediction error are then generated by randomly sampling values from the assumed normal distribution. When the ensemble mean error and spread are considered as the parameters of prediction errors, those parameters can be controlled by changing the values for the mean and variance of the normal distribution of prediction errors. The generation of an ensemble inflow prediction sequence is conducted by use of the following method. Firstly, a value is randomly sampled for the prediction error of an ensemble member for the next time step $(l=1)$. A series of prediction errors is then generated using a first-order autoregressive (AR(1)) model, which can be described as the following equations (Tokutsu et al., 2016):

$$
\begin{gathered}
e(l, m)=e^{\prime}(l, m)+\mu_{\mathrm{e}}(l) \\
e^{\prime}(l, m)=e^{\prime}(l-1, m) \cdot \rho_{\mathrm{L}} \cdot \frac{\sigma_{\mathrm{e}}(l)}{\sigma_{\mathrm{e}}(l-1)}+w(l, m) \sqrt{1-\rho_{\mathrm{L}}^{2}} \quad(2 \leq l \leq L)
\end{gathered}
$$

where $e(l, m)$ is prediction error of ensemble member $m(m=1, \ldots, M)$ provided for lead time $l(l=1, \ldots$, $L), \mu_{\mathrm{e}}(l)$ and $\sigma_{\mathrm{e}}(l)$ are respectively the mean and standard deviation of the normal distribution which errors of prediction for lead time $l$ follow, $\rho_{\mathrm{L}}$ is the serial correlation of prediction errors, and $w(l, m)$ is white noise which is randomly sampled from $\mathrm{N}\left(0,\left\{\sigma_{\mathrm{e}}(l)\right\}^{2}\right)$, respectively. The values for $\mu_{\mathrm{e}}(l)$ and $\sigma_{\mathrm{e}}(l)$ for each lead time are respectively estimated from the ensemble mean and spread of an operational ensemble hydrological prediction in this study. This can be considered as a natural extension of the generation method of a time series of single deterministic predictions (Takeuchi and Sivaarthitkul, 1995) to generate a time series of ensemble predictions.

If the probabilistic distribution of prediction errors is considered to have a long tail as it is often the case for the probabilistic distribution of a hydrological variable, one can consider a log-normal distribution. When a log-normal distribution with three parameters (LN3) is considered for the probabilistic distribution of prediction errors, equations for generation of predictions can be described as follows:

$$
\begin{gathered}
e(1, m)=\exp \left[\mu_{\mathrm{A}}(1)+\sigma_{\mathrm{A}}(1) \cdot \varepsilon(1, m)\right]+c_{\mathrm{A}}(1) \\
e(l, m)=\exp \left[\mu_{\mathrm{A}}(l)+\sigma_{\mathrm{A}}(l) \cdot e^{\prime}(l, m)\right]+c_{\mathrm{A}}(l) \quad(2 \leq l \leq L) \\
e^{\prime}(l, m)=\left[\varepsilon(l, m) \cdot \sqrt{1-\rho_{\mathrm{e}}(l, l-1)}\right]+\rho_{\mathrm{e}}(l, l-1) \cdot e^{\prime}(l-1, m)
\end{gathered}
$$




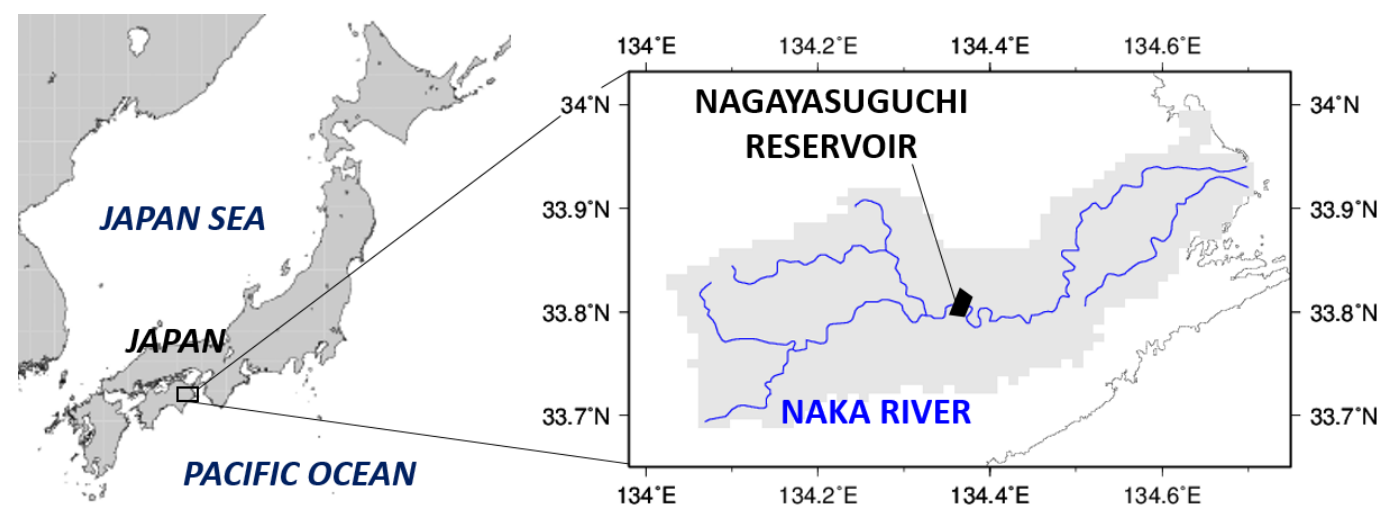

Figure 1: Location of the target reservoir and river basin

\begin{tabular}{lc}
\hline Items & Values \\
\hline Active storage capacity $\left(\mathrm{m}^{3}\right)$ & $43,497,000$ \\
Water use capacity $\left(\mathrm{m}^{3}\right)$ & $43,497,000$ \\
Flood control capacity $\left(\mathrm{m}^{3}\right)$ & $10,960,000$ \\
Maximal release discharge in case of non-flood condition $\left(\mathrm{m}^{3} / \mathrm{s}\right)$ & 500 \\
Designed inflow to start flood control $\left(\mathrm{m}^{3} / \mathrm{s}\right)$ & 2,500 \\
Designed maximal flood inflow $\left(\mathrm{m}^{3} / \mathrm{s}\right)$ & 6,500 \\
Designed maximal release discharge during flood control $\left(\mathrm{m}^{3} / \mathrm{s}\right)$ & 5,400 \\
\hline
\end{tabular}

Table 1: Storage volume allocation of the Nagayasuguchi Reservoir

$$
\rho_{\mathrm{e}}(l, l-1)=\frac{\log \left\{\rho_{\mathrm{L}}^{2}(l, l-1) \sqrt{\left[\exp \left(\sigma_{\mathrm{A}}^{2}(l)-1\right)\right] \cdot\left[\exp \left(\sigma_{\mathrm{A}}^{2}(l-1)-1\right)\right]}+1\right\}}{\sigma_{\mathrm{A}}(l) \cdot \sigma_{\mathrm{A}}(l-1)}
$$

where $\mu_{\mathrm{A}}(l), \sigma_{\mathrm{A}}(l)$ and $c_{\mathrm{A}}(l)$ are parameters of $\mathrm{LN} 3, \varepsilon(l, m)$ is a normal random number that follows $\mathrm{N}(0,1)$, respectively.

\section{Case Study}

\subsection{Target Reservoir and Simulation Settings}

The proposed method was applied to the Nagayasuguchi Reservoir in the Naka River basin, located in the south west part of Japan (Figure 1). Specs of the target reservoir are summarized as shown in Table 1. The reservoir has a catchment of $538.9 \mathrm{~km}^{2}$, and the river basin receives 2,000 to $3,000 \mathrm{~mm}$ of mean annual precipitation. All the storage of the reservoir is allocated for water use purpose in the non-flood condition, while some of that volume is also used for flood control capacity in the flood condition. The reservoir therefore needs to secure the empty volume of $10.96 \mathrm{MCM}$ by conducting preliminary release as a part of flood control operation in advance of flood arrival. The water storage volume must be decreased to $32.537 \mathrm{MCM}$ by conducting preliminary release if inflow of the reservoir is predicted to exceed $500 \mathrm{~m}^{3} / \mathrm{s}$. 

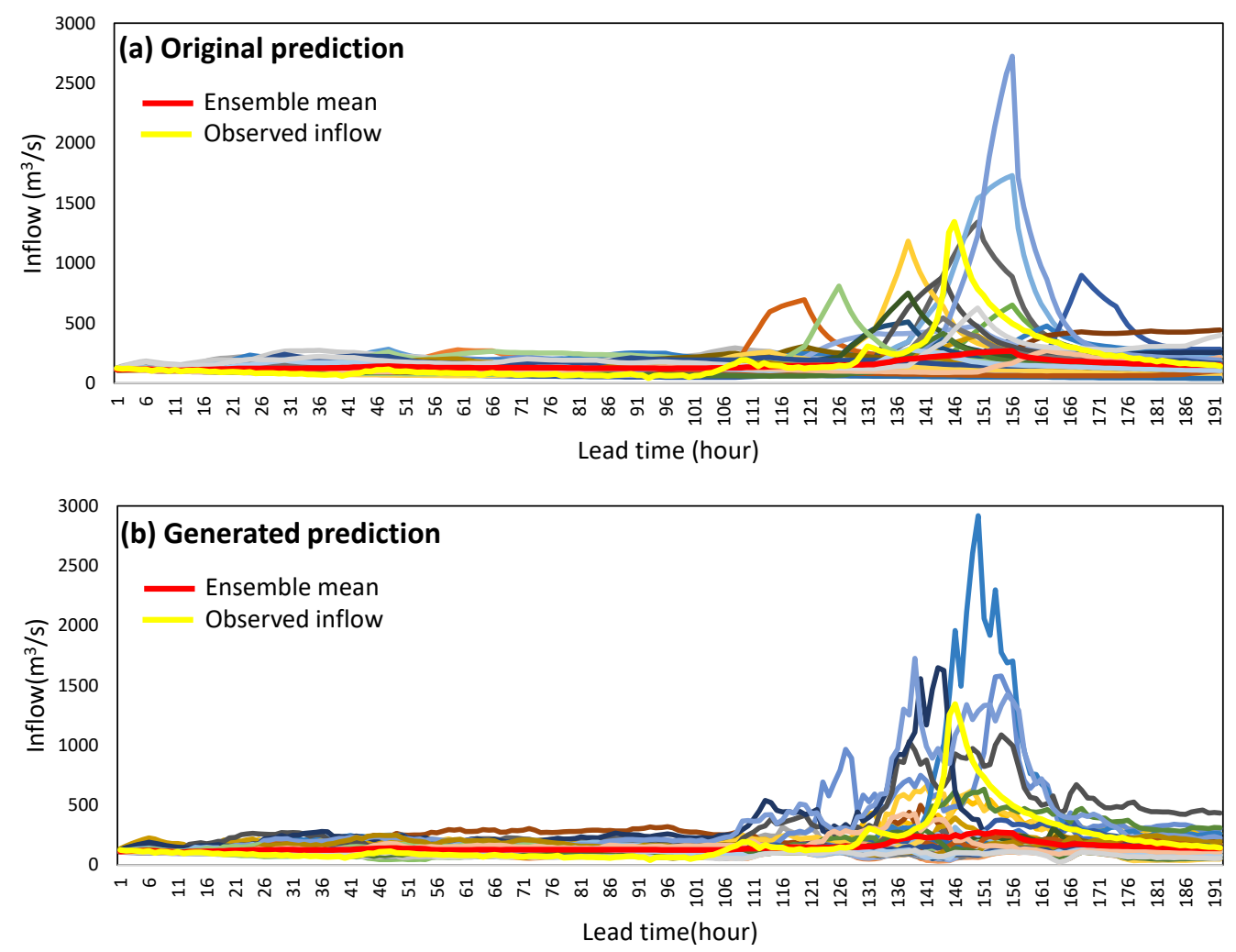

Figure 2: Ensemble inflow predictions: (a) estimated from operational forecast provided on 11 September, 2006, and (b) generated by the proposed model.

\subsection{Generation of Ensemble Inflow Predictions}

Results of generation of ensemble inflow predictions are shown in Figure 2 as an example. A LN3 was assumed as the probabilistic distribution of prediction errors in this example, and ensemble inflow prediction for the coming 192 hours was generated. The values of three parameters for the LN3 were estimated from ensemble prediction of Nagayasuguchi Reservoir's inflow derived from operational ensemble forecast of precipitation. One-week Ensemble Forecast of precipitation provided by Japan Meteorological Agency (JMA) was considered for the operational ensemble forecast of precipitation, and ensemble inflow prediction was estimated from this ensemble precipitation prediction by use of Hydro-BEAM (Hydrological River Basin Environment Assessment Model), a distributed rainfall-runoff model (Kojiri, 2006). It can be seen in Figure 2 that generated ensemble inflow prediction could simulate distributional characteristics of that estimated from JMA's Oneweek Ensemble Forecast of Precipitation (denoted as original prediction in Figure 2). Time series of each ensemble member is however slightly different between original and generated predictions.

\subsection{Monte Carlo Simulation of Reservoir Operation}

A case study was carried out to analyse effectiveness and risk of preliminary release operation of the target reservoir considering ensemble inflow prediction with given error characteristics. A normal 


\begin{tabular}{llllc}
\hline Scenario & Policy & $\begin{array}{l}\text { Rate of simulations where } \\
\text { preliminary release conducted } \\
(\%)\end{array}$ & $\begin{array}{l}\text { Rate of simulations where } \\
\text { water storage recovered }(\%)\end{array}$ & $\begin{array}{l}\text { Mean storage rate } \\
\text { after the flood (\%) }\end{array}$ \\
\hline 1 & 1 & 0.0 & 100.0 & 100.0 \\
1 & 2 & 10.3 & 100.0 & 100.0 \\
2 & 1 & 0.0 & 100.0 & 100.0 \\
2 & 2 & 99.7 & 96.7 & 99.9 \\
\hline
\end{tabular}

Table 2: Example of results of simulations on preliminary release operation

distribution was assumed for the probabilistic distribution of prediction errors in this analysis. The values of the error parameters $\mu \mathrm{e}(\mathrm{l})$ and $\sigma \mathrm{e}(1)$ (in Equations (1) and (2)) for the generation of ensemble inflow predictions were respectively decided from the results of analysis on the error characteristics of ensemble inflow predictions for the coming 192 hours derived from JMA's One-week Ensemble Forecast of precipitation by use of Hydro-BEAM. A thousand of ensemble inflow predictions were generated, and a thousand of simulations on preliminary release and flood control operations was conducted considering each generated ensemble inflow predictions as a Monte Carlo simulation.

The example of results of simulations is shown in Table 2. The results of simulations when a historical flood event with the peak inflow rate of $535.0 \mathrm{~m}^{3} / \mathrm{s}$ was considered are shown in Table 2 . Two policies were applied to consider ensemble inflow predictions: The policy where ensemble mean predictions were considered (denoted by Policy 1 ), and the policy where the ensemble member with the maximum value of prediction was considered (denoted by Policy 2). The effects of preliminary release operation were also analysed when the degree of ensemble mean errors were two-thirds as small as the original degree (denoted by the Scenario 2), in addition to the simulation when the value of ensemble mean errors estimated from operational inflow predictions (denoted by Scenario1). It can be seen here that preliminary release operation was not successfully conducted in all simulations when ensemble mean predictions were considered to decide to start preliminary release. This is because ensemble mean predictions basically had a tendency of underestimation in operational ensemble inflow predictions and could not foresee the peak value of inflow well. On the other hand, the number of simulations where preliminary release was successfully conducted was slightly increased if the ensemble member with the maximal value of prediction was considered (Policy 2). The rate of success in preliminary release increased drastically when the ensemble mean error was improved (Scenario 2) with Policy 2. Although a minor drawback can be seen in water recovery of the reservoir after the flood when Policy 2 was taken in Scenario 2, this option can be considered as a balanced way to conduct preliminary release operation considering ensemble inflow predictions in Scenario 2. In this way, the proposed method can provide quantitative information on effectiveness or risks of preliminary release operation considering ensemble hydrological predictions, which can be useful to decide a policy how to consider those ensemble predictions, or to show how far the prediction model should be improved in order to provide reliable decision for preliminary release operation.

\section{Conclusion}

A Monte Carlo-based method to analyse effectiveness and risks of integrated operation of a multipurpose reservoir for flood management considering real-time ensemble hydrological predictions is developed. Through the case study, it was shown that the method can provide quantitative information on effectiveness or risks of preliminary release operation considering ensemble hydrological predictions, which can be useful to decide a policy how to consider those ensemble predictions. The analysis can also contribute to identify how good the prediction model should be in order to give a 
reliable decision for preliminary release when the ensemble hydrological predictions provided by the model are considered.

\section{Acknowledgement}

This work was supported by JSPS KAKENHI Grant Number 16K06510. Data of the target reservoir and river basin were provided by Japan Ministry of Land, Infrastructure, Transport and Tourism. The authors would like to express sincere appreciation to all of them.

\section{References}

Faber, B.A. and Stedinger, J. (2001). Reservoir optimization using sampling SDP with ensemble streamflow prediction (ESP) forecasts, Journal of Hydrology, 249, 113-133.

Kim, Y.O., Eum, H.I., Lee, E.G. and Ko, I.H. (2007). Optimizing operational policies of a Korean multireservoir system using sampling stochastic dynamic programming with ensemble streamflow prediction, Journal of Water Resources Planning and Management, 133(1), 4-14.

Kojiri, T. (2006). Hydrological river basin assessment model (Hydro-BEAM). Watershed Models, Singh, V.P. \& Frevent, D.K. (eds). Taylor \& Francis, CRC Press: Boca Raton, FL, USA, 613-626.

Masuda, H. and Oishi, S. (2013). Study on optimization of the integrated operation of dams using ensemble prediction in the upper reaches of the Nabari river, Proc. 35th IAHR World Congress, S10065.

Nohara, D., Saito, H. and Hori, T. (2017). A framework to assess effectiveness and risks of integrated reservoir operation for flood management considering ensemble hydrological prediction, Proc. XVI World Water Congress, Cancun, ABSID359 (14pp.).

Takeuchi, K. and Sivaarthitkul, V. (1995). Assessment of effectiveness of use of inflow forecasts to reservoir management, IAHS Publications, 231, 299-309.

Tokutsu, M., Nohara, D. and Hori, T. (2016). A Monte Carlo-based method to analyze effectiveness of reservoir operation for water supply considering imperfect ensemble inflow prediction, Proc. of the 7th International Conference on Water Resources and Environment Research, S07-02 (6pp). 